\title{
Low-Cost Broadband Electronic Coupler for Estimation of Radiated Emission of Integrated Circuits in TEM Cell
}

\author{
A. Boyer, S. Ben Dhia
}

\begin{abstract}
TEM cell is a well-known method for the qualification of radiated electromagnetic emission from integrated circuits. Combined with a $180^{\circ}$ hybrid coupler, it provides a simple method for quantifying radiated emission. However, typical transformer or coupled line-based hybrid couplers have limited bandwidth which cannot cover the full frequency range required by integrated circuit emission tests $(150 \mathrm{kHz}$ to $1 \mathrm{GHz})$. This paper presents the design and the validation of a low-cost broadband electronic version of a $180^{\circ}$ hybrid coupler dedicated to the estimation of radiation emission from TEM cell measurements. The proposed prototype is able to cover a large frequency range (from DC to $1 \mathrm{GHz}$ ) with a sufficient sensitivity and dynamic range for radiated emission tests in TEM cell. The cost of the presented coupler is very reasonable as it is entirely based on commercial opamps.
\end{abstract}

Index Terms - Integrated Circuit, TEM cell, radiated emission measurement

\section{INTRODUCTION}

$\mathrm{T}$ EM cell method, known also as IEC61967-2 [1], is a popular test for the characterization of the radiated emission (RE) of integrated circuits (IC) due to its compact size and its good sensitivity compared to other RE test facilities (e.g. open area test site or anechoic chamber). The typical set-up of TEM cell RE measurement is presented in Fig. 1.a. The TEM cell is a rectangular stripline structure whose dimensions have been defined to ensure $50 \Omega$ matching and transverse electromagnetic (TEM) mode propagation up to $1.2 \mathrm{GHz}$. Any small powered device placed within the cell excites its TEM mode and induces a voltage on both terminals, which is usually measured by a spectrum analyser.

Actually, the relationship between the measured voltage and $\mathrm{RE}$, for example in free space far-field conditions, is not straightforward. If the excitation source is electrically small and placed at the cell center $(z=0)$, RE can be estimated according to the method described in [2], which is illustrated in Fig. 1.b. The excitation source (here the IC under test) can be expanded into equivalent electric and magnetic dipole moments $P_{Y}$ and $M_{X}$ whose RE is easily calculable. They contribute respectively to electric $(\mathrm{E})$ and magnetic-field $(\mathrm{H})$

A. Boyer, S. Ben Dhia are with LAAS-CNRS, 7 avenue du colonel Roche, F-31077 Toulouse Cedex 4, France (e-mail: alexandre.boyer@laas.fr, sonia.ben.dhia@laas.fr).
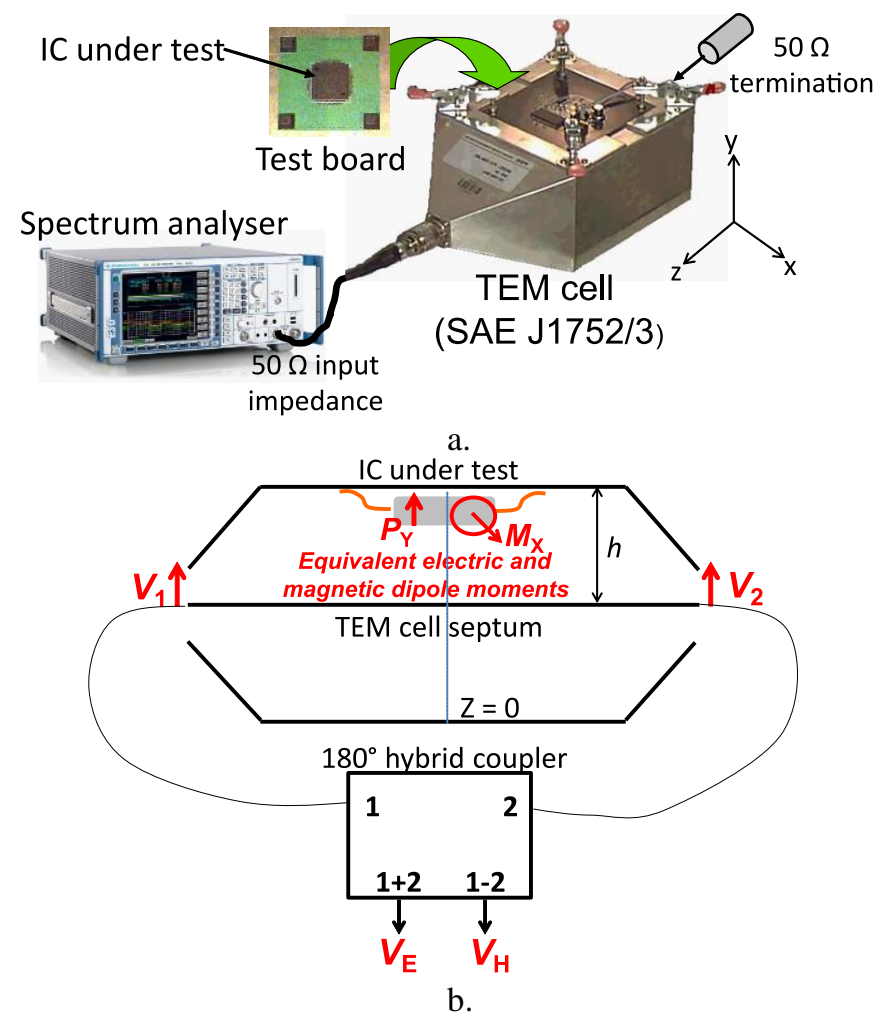

Fig. 1. a. Typical IC radiated emission measurement set-up; b. Extraction of the electric and magnetic-field coupling induced voltages

coupling induced voltages in TEM cell, noted $V_{\mathrm{E}}$ and $V_{\mathrm{H}}$. The TEM cell termination voltages, $V_{1}$ and $V_{2}$, are then obtained according to (1) and (2), where $k_{0}$ is the propagation constant, $Z_{\mathrm{C}}$ the TEM cell characteristic impedance and $h$ the distance between the IC under test and the TEM cell septum.

$$
\begin{aligned}
& V_{1}=\frac{1}{2}\left(P_{Y}+j k_{0} M_{X}\right) \frac{Z_{C}}{h}=\frac{1}{2}\left(V_{E}+V_{H}\right) \\
& V_{2}=\frac{1}{2}\left(P_{Y}-j k_{0} M_{X}\right) \frac{Z_{C}}{h}=\frac{1}{2}\left(V_{E}-V_{H}\right)
\end{aligned}
$$

Measuring the voltages $V_{\mathrm{E}}$ and $V_{\mathrm{H}}$ is essential to extract the equivalent electric and magnetic dipole moments. From these figures, not only the RE due to the IC in far-field, but also in 
near-field regions, can be accurately estimated. A typical experimental method to extract $V_{\mathrm{E}}$ and $V_{\mathrm{H}}$ relies on the use of a $180^{\circ}$ hybrid coupler as presented in [3], [4] for example. The method is illustrated in Fig. $1 b$. Depending on the frequency range, they are based on transformers, coupled transmission lines or waveguides. However, they never cover the full frequency range required by IEC61967 $(150 \mathrm{kHz}$ to $1 \mathrm{GHz})$, since they usually cover one or two decades of frequency. Moreover, they are expensive devices whose typical costs exceed several hundreds of euros. Surprisingly, in spite of its simplicity, no solution based on active devices has been proposed, certainly because of the required frequency and sensitivity constraints. However, low-cost high-frequency opamps are available whose frequency and noise characteristics comply with TEM cell test requirements. The purpose of this letter is to present the design and the characterization of a wideband home-made electronic coupler dedicated to the estimation of radiated emission of ICs from TEM cell measurements. Compared to conventional $180^{\circ}$ hybrid couplers, the proposed coupler covers a large frequency range (from DC to $1 \mathrm{GHz}$ ) which complies with IEC61967 requirements at a reasonable price.

\section{ELECTRONIC COUPLER DESIGN}

The proposed coupler is a basic adder and substractor circuit based on the AD8000 op-amp from Analog Devices [5]. This high-speed current feedback amplifier is dedicated to IF/RF gain stage or high-resolution video graphic applications. With a small-signal bandwidth of $1.5 \mathrm{GHz}$, a high slew rate of 4100 $\mathrm{V} / \mu \mathrm{s}$, a low input voltage noise $(1.6 \mathrm{nV} / \sqrt{\mathrm{Hz}}$ above $100 \mathrm{kHz})$, a low distortion (spurious-free dynamic range of $62 \mathrm{dBc}$ at 50 $\mathrm{MHz}$ ) and an excellent stability, it is a good candidate for the processing of voltage measured at TEM cell terminals.

A home-made prototype has been designed on a two-layer printed-circuit board (PCB). The PCB has not been mounted within a shielded cabinet. The overall cost of the design and fabrication of one prototype is $90 €$, which makes it an affordable measurement device. The electrical schematic of the proposed coupler is presented in Fig. 2. Although its principle is simple, a careful design is required. The length of PCB traces must be limited and keep symmetrical to reduce parasitic output capacitance which may compromise stability and limit phase error. IEC61967-2 standard requires a voltage standing wave ratio (VSWR) less than 1.5:1, i.e. an input reflection coefficient less than $-14 \mathrm{~dB}$. SMA coaxial connectors are mounted at input and output terminals. The input impedance of the coupler must be controlled to ensure sufficient $50 \Omega$ matching, because of the capacitive behaviour of the op-amp inputs (typically $3.6 \mathrm{pF}$ ).

The choice of resistors is a compromise between stability, gain flatness and minimization of noise. In this design, identical $330 \Omega$ resistors are used so that the overall gain of the coupler is equal to 1 . The output noise is estimated to $17 \mathrm{nV} / \sqrt{\mathrm{Hz}}$ above $100 \mathrm{kHz}$, i.e. $-5 \mathrm{~dB} \mu \mathrm{V}$ with $1 \mathrm{kHz}$ RBW receiver, which is equivalent to noise floor of typical spectrum analyser. As the gain of the input stage of the coupler is equal to 2 and the maximum output voltage swing of the op-amp is $2 \mathrm{~V}$, the largest measurable signal at the input is equal to $1 \mathrm{~V}$. The dynamic range between the largest and lowest harmonics of the input signal is limited by the distortion introduced by

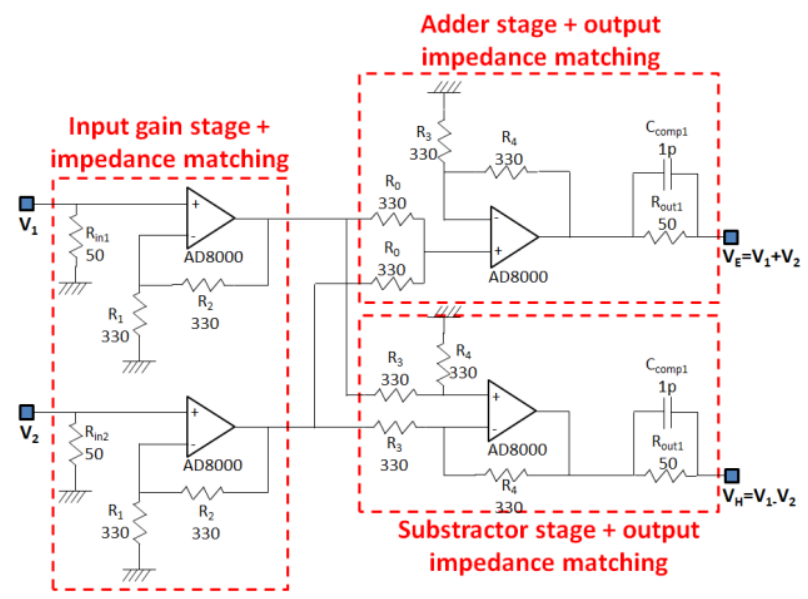

Fig. 2. Schematic of the proposed electronic coupler

the op-amp. Below $100 \mathrm{MHz}$, the dynamic range exceeds 50 dB. As typical IC emission levels seldom exceed $50 \mathrm{~dB} \mu \mathrm{V}$, the dynamic range and the noise of the proposed electronic coupler comply with the requirements of the TEM cell measurement. This coupler has also other advantages compared to transformer or stripline-based hybrid couplers: it provides an excellent isolation between output terminals and there is no coupling loss between input and output. Moreover, the gain can be adjusted and increased, but it results in a reduced cut-off frequency.

\section{EXPERIMENTAL CHARACTERIZATION}

The performances of the designed prototype have been characterized. In order to prevent external interference issues, all the tests have been made in a Faraday cage. The reflection coefficients of both inputs have been measured, as shown in Fig. 3. They are less than $-20 \mathrm{~dB}$ except between $800 \mathrm{MHz}$ and $1.2 \mathrm{GHz}$, but they do not exceed the $-14 \mathrm{~dB}$ requirement.

The noise introduced by the coupler has been characterized by measuring the noise floor of the spectrum analyser (Keysight N9341C) with and without the electronic coupler. Without the coupler, with a resolution bandwidth of $1 \mathrm{kHz}$, the noise floor of the spectrum analyser is $-5 \mathrm{~dB} \mu \mathrm{V}$. With the coupler, it rises about $4 \mathrm{~dB}$. If we suppose that the noise voltages produced by the spectrum analyser and the coupler are gaussian and uncorrelated, the amplitude of the noise introduced by the coupler can be estimated to $-3.3 \mathrm{~dB} \mu \mathrm{V}$, which is acceptable for typical TEM cell emission measurements.

Correct sum and difference operations requires a small gain and phase differences between both signal channels. It depends on the design symmetry. The gain difference between signal channels is less than $2 \mathrm{~dB}$ up to $1 \mathrm{GHz}$, while the phase difference is less than $20^{\circ}$. Fig. 4 illustrates the frequency response of the sum and difference channel. The $-6 \mathrm{~dB}$ cut-off frequency is reached at $1.01 \mathrm{GHz}$ and $1.1 \mathrm{GHz}$. The gain is constant from DC to $100 \mathrm{MHz}$ and varies in the range 100 


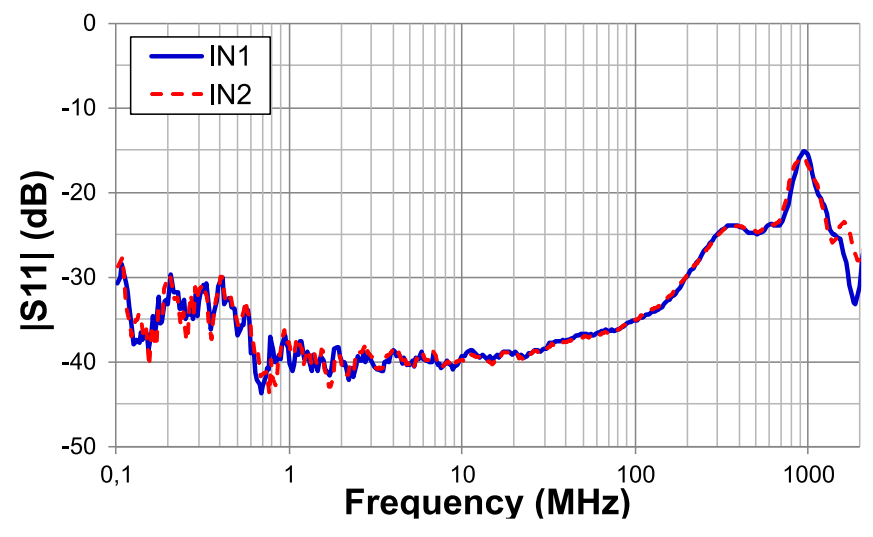

Fig. 3. Characterization of the reflection coefficient of coupler inputs

$\mathrm{MHz}$ to $1000 \mathrm{MHz}$, according to the choice of resistor values and an optional compensation output capacitor. Only a compromise between gain flatness and bandwidth can be found. However, the gain fluctuation can be characterized for calibration purpose.

\section{VALIDATION CASE}

In order to validate the results delivered by our electronic coupler, the $\mathrm{E}$ and $\mathrm{H}$ field coupling induced voltages $V_{\mathrm{E}}$ and $V_{\mathrm{H}}$ are measured with a spectrum analyser (Keysight N9341C). The device under test is a $75 \mathrm{~mm}$ long $50 \Omega$ microstrip line excited by a $10 \mathrm{MHz}$ square signal with $4 \mathrm{~ns}$ transition time and a duty cycle of $50 \%$. The line ends are terminated by either 50 $\Omega$ load, shorted or opened. Depending on the line loading, electric, magnetic field, or both fields are predominant in nearfield region. For comparison purpose, measurements are also performed with a commercial $180^{\circ}$ hybrid coupler (model ET Industries J-053-180) whose bandwidth is $500 \mathrm{MHz}$ to 3000 MHz. Finally, measurement results are compared with the predicted values of $\mathrm{V}_{\mathrm{E}}$ and $\mathrm{V}_{\mathrm{H}}$. The prediction relies on (3) and (4) [6], which gives the electric and magnetic field coupling due to the equivalent electric and magnetic dipole moments $P_{Y}$ and $M_{X}$ of the line which is supposed electrically short.

$$
\begin{aligned}
& V_{E}(f)=P_{Y}(f) \frac{Z_{C}}{h}=j 2 \pi f c_{\text {line }} V_{\text {line }}(f) \frac{Z_{C} l_{\text {line }} h_{\text {line }}}{h} \\
& V_{H}(f)=j \frac{2 \pi f}{c_{0}} M_{X}(f) \frac{z_{C}}{h}=j \frac{2 \pi f}{c_{0}} I_{\text {line }}(f) \frac{Z_{C} l_{\text {line }} h_{\text {line }}}{h}
\end{aligned}
$$

where $f$ is the frequency, $l_{\text {line }}$ and $h_{\text {line }}$ are the length and the height of the microstrip line, $c_{\text {line }}$ its per-unit-length capacitance, and $V_{\text {line }}$ and $I_{\text {line }}$ the measured excitation voltage and current of the line. A Scilab script was developed to compute $V_{\mathrm{E}}$ and $V_{\mathrm{H}}$ voltages. The model was previously validated by comparing simulation results with S-parameter measurements between the line under test and the TEM cell. A good agreement was observed up to $1 \mathrm{GHz}$.

Initially, the line under test is terminated by a $50 \Omega$ load. Measurement and simulation results of $V_{\mathrm{E}}$ and $V_{\mathrm{H}}$ voltages are compared in Fig. 5. For readability, only the envelop of emission spectra are plotted, which presents the maximum

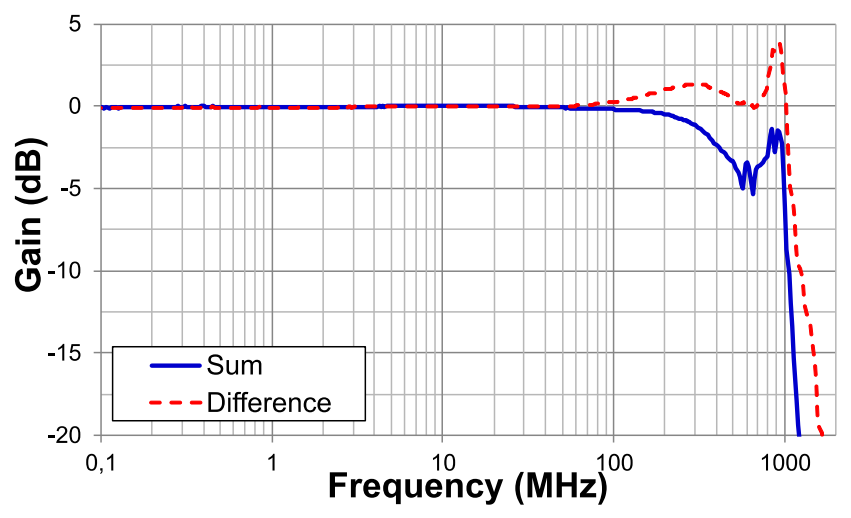

Fig. 4. Characterization of the gain of the sum and difference channels

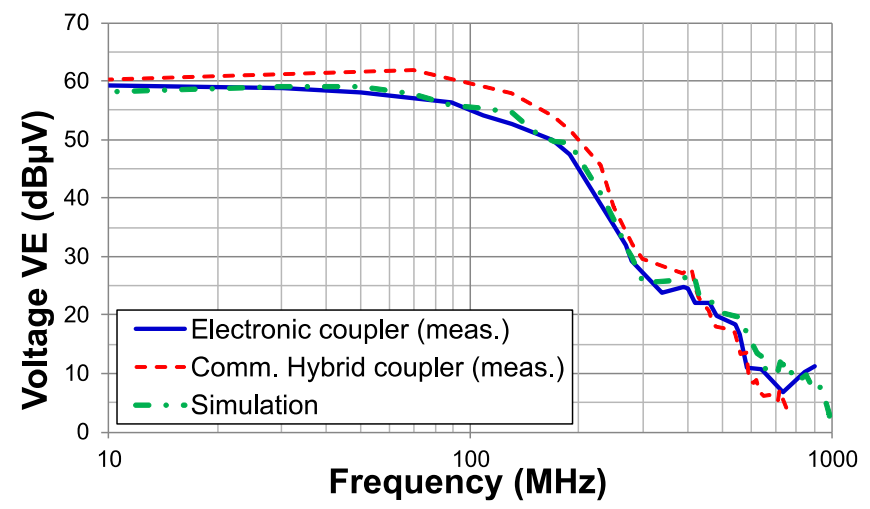

(a)

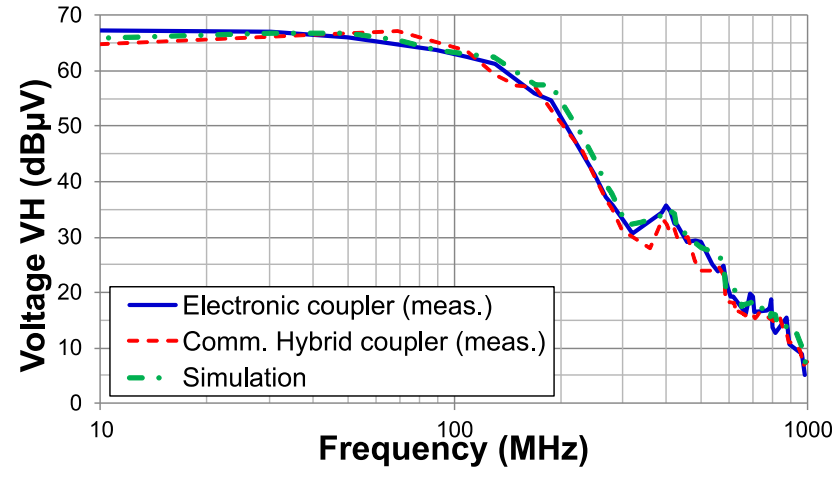

(b)

Fig. 5. Line under test terminated by $50 \Omega$. Comparison between measurements with the proposed electronic coupler, a commercial $180^{\circ}$ hybrid coupler and simulation of: (a) E-field coupling induced voltage $V_{\mathrm{E}}$, (b) H-field coupling induced voltage $V_{\mathrm{H}}$

values taken by the spectrum. The measurements done with the proposed coupler fit with simulation, proving the validity of the measurements done with the proposed coupler up to $1 \mathrm{GHz}$. They also fit with the measurement done with the commercial $180^{\circ}$ hybrid coupler in the range $500 \mathrm{MHz}$ to $1 \mathrm{GHz}$.

Two other configurations are tested: either the line under test end is opened to enhance $\mathrm{E}$ field and limit $\mathrm{H}$ field generation, or the line under test end is shorted to enhance $\mathrm{H}$ field and limit $E$ field generation. Measurement and simulation results of $V_{\mathrm{E}}$ and $V_{\mathrm{H}}$ voltages are compared in Fig. 6. In the top figure, the line under test is shorted and the $\mathrm{E}$ field coupling induced voltage is captured. In low frequency, as long as the 


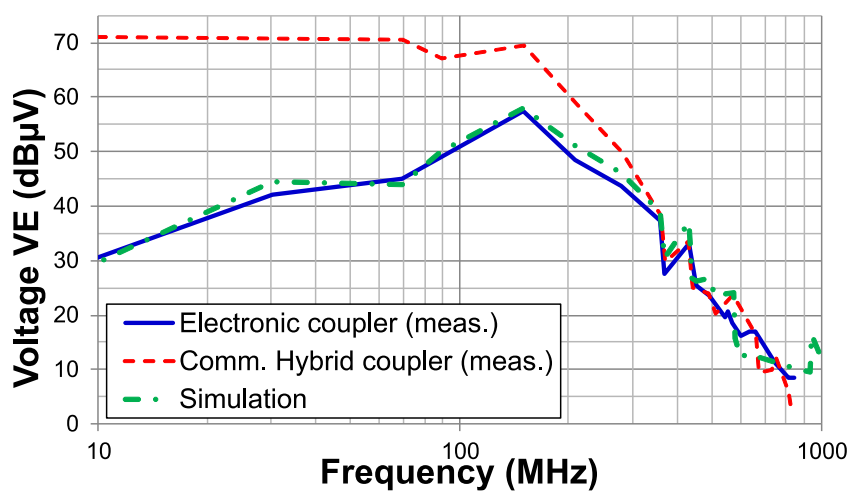

(a)

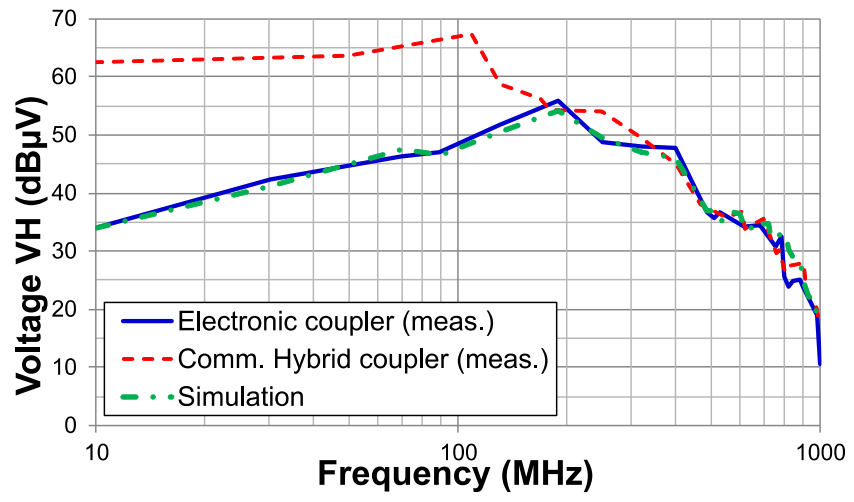

(b)

Fig. 6. Comparison between measurements with the proposed electronic coupler, a commercial $180^{\circ}$ hybrid coupler and simulation of: (a) Line under test terminated by a short. E-field coupling induced voltage $V_{E}$, (b) Line under test opened at its end. H-field coupling induced voltage $V_{H}$

line is electrically small, the voltage $V_{E}$ remains negligible as shown by the measurement done with the proposed electronic coupler and the simulation, which are in good agreement. In this situation, results provided by the commercial coupler are false up to $300 \mathrm{MHz}$ since it is used outside its nominal frequency range. Above $500 \mathrm{MHz}$, measurements made with both couplers are also in agreement. Fig. 6 (bottom) presents similar results for the voltage $V_{\mathrm{H}}$ when the end of the line is opened. The induced voltage due to magnetic field coupling remains negligible in low frequency. Measurements with the proposed electronic coupler are in good agreement with the simulation results, and with the measurement results obtained with the commercial $180^{\circ}$ hybrid coupler between $500 \mathrm{MHz}$ and $1 \mathrm{GHz}$.

\section{CONCLUSION}

In this letter, an electronic version of a $180^{\circ}$ hybrid coupler has been designed in order to separate the contributions of electric and magnetic field coupling in emission measurement of ICs in TEM cell. This type of measurement is essential to estimate the radiated emission of ICs not only in far-field but also in near-field conditions. Compared to typical transformer or coupled line-based hybrid coupler, the proposed coupler relies on low-cost off-the-shelf op-amps. It covers all the frequency range required by the emission standard test IEC61967-2 (150 kHz to $1 \mathrm{GHz})$, without any excessive degradation of noise floor of the measurement receiver.

\section{REFERENCES}

[1] IEC61967-2: Integrated Circuits - Measurement of Electromagnetic Emissions, $150 \mathrm{kHz}$ to $1 \mathrm{GHz}$ - Part 2: Measurement of Radiated Emissions - TEM Cell and Wideband TEM Cell Method, International Electrotechnical Commission, 2005, IEC.

[2] P. Wilson, "On Correlating TEM Cell and OATS Emission Measurements", IEEE Trans. on EMC, vol. 37, no. 1, Feb. 1995, pp. 116, doi: 10.1109/15.350235.

[3] Kasturi V., Deng S., Hubing T., Beetner D., "Quantifying Electric and Magnetic Field Coupling from Integrated Circuits with TEM Cell Measurements," IEEE Int. Symp. on EMC, Portland, USA, August 2006, pp. 422-425, DOI: 10.1109/ISEMC.2006.1706339.

[4] R. Blecic, H. Stimac, R. Gillon, B. Nauwelaers, A. Baric, "Improved Estimation of Radiated Fields of Unintentional Radiators by Correction of the Impedance Mismatch Between a Transverse Electromagnetic Cell and a Hybrid Coupler", IEEE Trans. on EMC, vol. 60, no. 6, Feb. 1995, pp. 1717-1725, DOI: 10.1109/TEMC.2017.2769021.

[5] AD8000, 1.5 GHz, Ultrahigh Speed Op Amp, Datasheet, Rev. C, Analog Devices, 2016, www.analog.com.

[6] A. Boyer, E. Sicard, "Basis of Electromagnetic Compatibility of Integrated Circuits", Presses Universitaires du Midi, Toulouse, France, 2017, ISBN: 978-2-8107-0522-1.

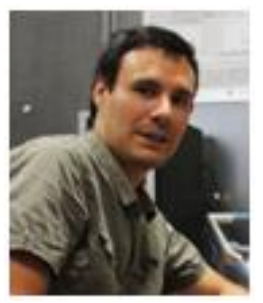

Alexandre Boyer obtained a Masters degree in electrical engineering in 2004 and $\mathrm{a} \mathrm{PD}$ in Electronics from the Institut Nationale des Sciences Appliquées (INSA) in Toulouse, France, in 2007. He is currently an Associate Professor in the Department of Electrical and Computer Engineering at INSA, Toulouse. He is leading his research at the Laboratoire d'Analyse et d'Architecture des Systèmes (LAAS-CNRS), as part of the 'Energy and Embedded Systems' research group. His current research interests include EMC measurements, IC EMC and reliability modeling, and computer aided design (CAD) tool development for EMC (IC-EMC freeware).

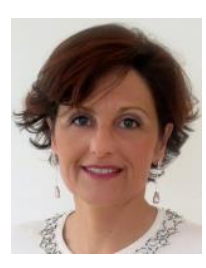

Sonia Ben Dhia obtained her Masters degree in electrical engineering in 1995, and a Ph.D. in Electronic Design from the Institut National des Sciences Appliquées (INSA), Toulouse, France, in 1998. Full professor at INSAToulouse (French engineering institute), Department of Electrical and Computer Engineering, she teaches digital electronics, IC testability and reliability, and analog and RF CMOS design. Her research interests at LAAS - CNRS Toulouse include signal integrity in nano-scale CMOS ICs, electromagnetic compatibility and reliability of ICs. She has authored and co-authored 3 books, more than 120 publications in peer-reviewed journals \& conference proceedings and supervised $14 \mathrm{PhD}$ theses and 9 M.Sc. theses. 